\title{
Secondary organising pneumonia complicating acute respiratory distress syndrome caused by severe influenza A: a case report
}

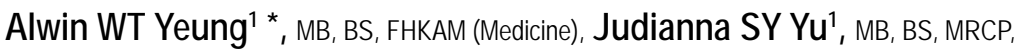 \\ Richard WC Wong ${ }^{2}$, FHKCPath, FHKAM (Pathology) \\ ${ }^{1}$ Department of Medicine and Geriatrics, Ruttonjee Hospital, Wan Chai, Hong Kong \\ ${ }^{2}$ Department of Clinical Pathology, Pamela Youde Nethersole Eastern Hospital, Chai Wan, Hong Kong \\ *Corresponding author: yeungwt@ha.org.hk \\ Hong Kong Med J 2020;26:139-41 \\ https://doi.org/10.12809/hkmj197918
}

\section{Case report}

A 69-year-old man with a history of diabetes mellitus and benign prostatic hypertrophy was admitted to our medical unit with a 1-week history of fever, runny nose, and shortness of breath. Chest X-ray revealed bilateral diffuse ground glass opacities in both lung fields. The patient had type 1 respiratory failure and required non-invasive positive pressure ventilation to maintain oxygenation. The patient was given ceftriaxone 1 g every 12 hours, and doxycycline $100 \mathrm{mg}$ and oseltamivir $75 \mathrm{mg}$ twice daily. He was transferred to the intensive care unit and intubated due to severe acute respiratory distress syndrome (ARDS). The patient's nasopharyngeal swab was tested by polymerase chain reaction and the result was positive for influenza A virus H1 RNA. His clinical course was further complicated by septic acute kidney injury that necessitated continuous venovenous haemofiltration. His lungs gradually improved with supportive measures including prone ventilation and muscle paralysis for 48 hours. The fractional oxygenation requirement improved from 1.0 to 0.4 between day 1 and day 8 of admission and chest X-ray showed improving aeration of both lungs.
Unfortunately, his ventilatory requirement deteriorated again from day 9 onwards, with chest $\mathrm{X}$-ray showing new right upper zone and left middle zone infiltrates (Fig 1). Repeat microbiological investigation results were negative except for Candida albicans and Candida species in the endotracheal culture. Antibiotics were upgraded to meropenem $500 \mathrm{mg}$ every 8 hours. High-resolution computed tomography of the thorax on day 14 showed patchy consolidation with air bronchogram and ground glass opacities in both lungs, more prominent in the right upper lobe, right lower lobe, and left lower lobe, and mild bilateral pleural effusions.

Bronchoscopy with transbronchial biopsy of the apical segment of the left lower lobe was performed uneventfully. Histological examination of the biopsy specimen revealed alveolar lining partially composed of cuboidal cells with foamy cytoplasm, a variable degree of nuclear enlargement and pleomorphism, and prominent nucleoli. The histology could be suggestive of adenocarcinoma with appropriate clinical settings. In view of the unusual clinical course, it was decided to repeat

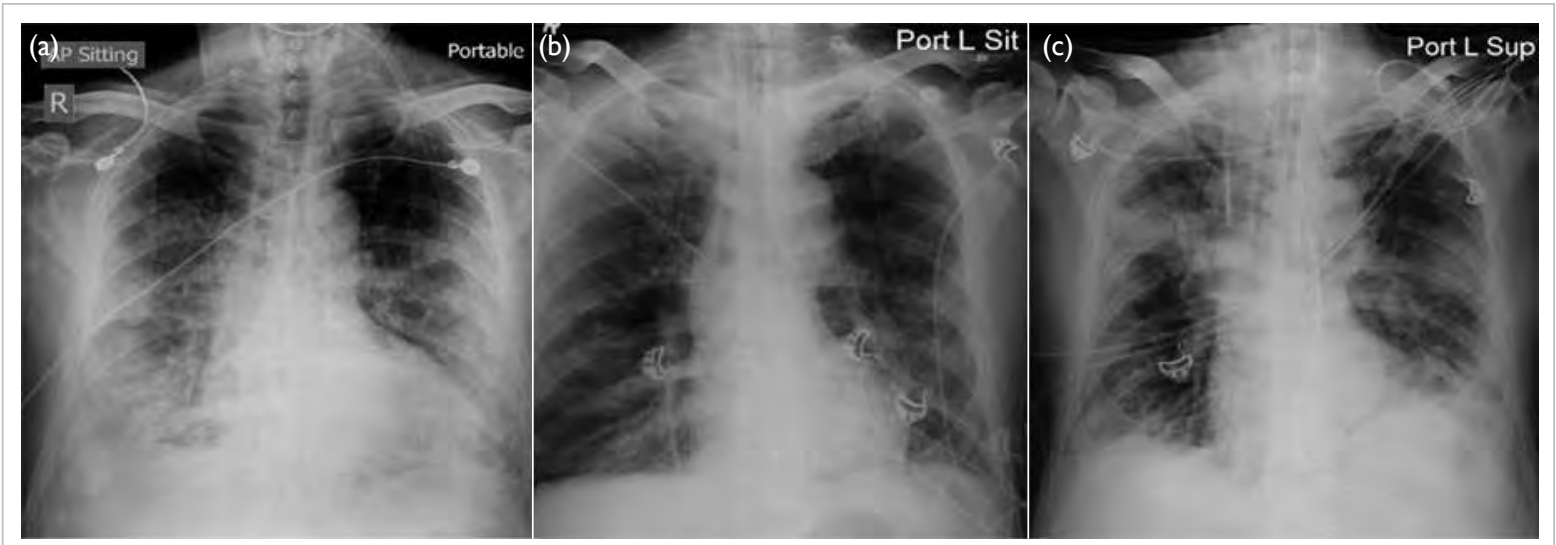

FIG I. A 69-year-old man with respiratory failure. Chest X-rays on (a) day I, (b) day 8, and (c) day 9 showing initial improving bilateral ground glass opacities from day I to day 8 and new right upper zone and left middle zone infiltrates on day 9 
transbronchial biopsies at different sites, namely at the left lower lobe apical segment again on day 18 and the right upper lobe posterior segment on day 19. Both histological specimens revealed a pattern compatible with organising pneumonia (OP) [Fig 2]. Intravenous hydrocortisone $100 \mathrm{mg}$ every 8 hours and subsequently enteral prednisolone $40 \mathrm{mg}$ daily were prescribed, and surgical tracheostomy performed in view of the prolonged mechanical ventilation.

The patient showed dramatic improvement with steroid treatment. He was weaned off mechanical ventilatory support and renal replacement therapy and discharged from the intensive care unit on day 22. He underwent rehabilitation in a general ward and was weaned off tracheostomy and supplementary oxygen. Serial chest X-rays showed resolution of both lung consolidations with minimal residual fibrotic scarring.

\section{Discussion}

In general, $\mathrm{H} 1$ influenza infection causes mild upper respiratory tract symptoms. A minority of patients present with ARDS secondary to viral pneumonia with or without bacterial co-infection. Apart from neuraminidase inhibitors such as oseltamivir management is mostly supportive. This includes protective lung ventilation and a conservative fluid management strategy. In moderate to severe ARDS with partial pressure of oxygen/fraction of inspired oxygen ratio $<150 \mathrm{~mm} \mathrm{Hg}, 48$ hours of muscle

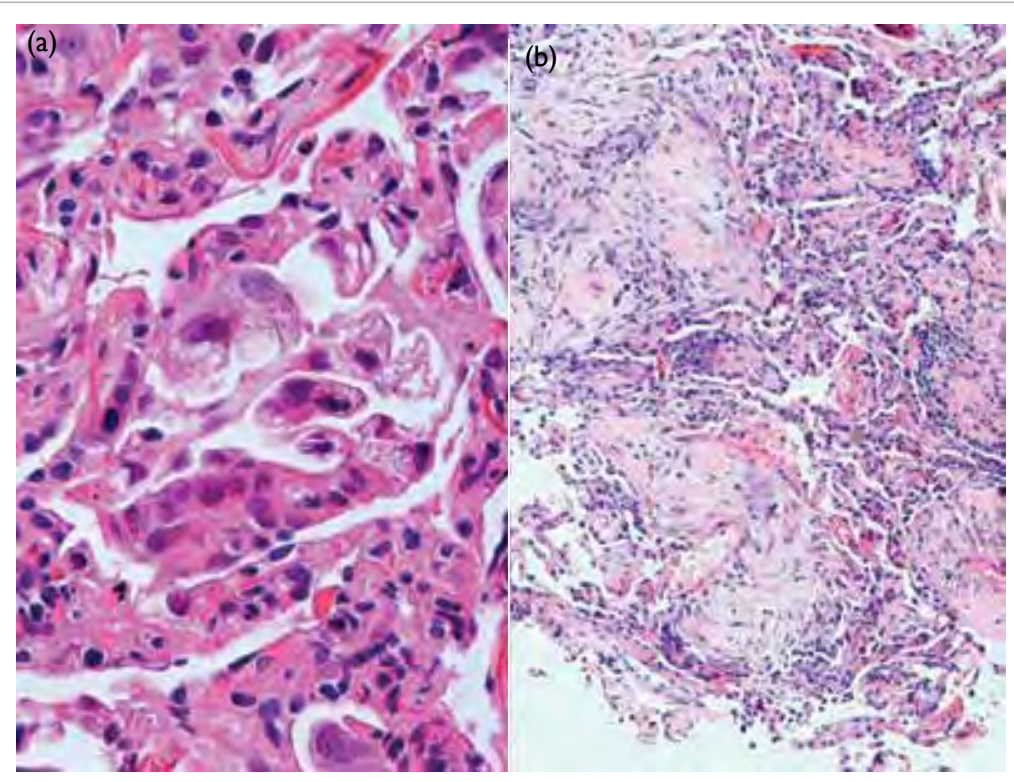

FIG 2. (a) From the same 69-year-old man with respiratory failure, initial transbronchial biopsy of the apical segment of the left lower lobe on day 14 showing pneumocytes with florid reactive nuclear atypia and foamy cytoplasm (haematoxylin and eosin, $\times 400$ ). (b) A typical organising pneumonia pattern is evident in the subsequent biopsy of the posterior segment of the right upper lobe (haematoxylin and eosin, $\times 100$ ) paralysis, prone ventilation, and extracorporeal membrane oxygenation may be required.

The definition of ARDS includes acute onset within 1 week, bilateral radiological opacities not explained by pleural effusion or lung collapse, respiratory failure not explained by heart failure, or fluid overload with partial pressure of oxygen/ fraction of inspired oxygen ratio $<300 \mathrm{~mm} \mathrm{Hg}$ under a positive end-expiratory pressure of at least $5 \mathrm{~cm}$ $\mathrm{H}_{2} \mathrm{O}$. Typically, patients with ARDS exhibit diffuse alveolar damage in histological specimens, divided into an acute exudative phase shortly after the pulmonary insult, followed by an organising phase, with or without a final fibrotic phase. However, a continuum and overlapping features exist, especially late in the first week or if the patient has encountered repeat pulmonary insults. The acute exudative phase is characterised by hyaline membranes that gradually disappear in the subsequent organising phase. The organising phase is characterised by interstitial fibrosis and pronounced type 2 pneumocyte hyperplasia. Cytological atypia may be quite pronounced and can be confused with malignancy, as in our case. ${ }^{1}$ Subsequently, diffuse alveolar damage will gradually resolve although some may progress to a fibrotic phase with continued interstitial fibrosis and compromises lung function.

Other histological patterns can present in patients with ARDS, including OP and acute fibrinous OP. In acute fibrinous OP, the alveolar spaces are filled with organising fibrin balls instead of hyaline membranes, whereas in OP, patchy accumulation of intra-alveolar organising fibroblastic tissue primarily centred around bronchioles is present. ${ }^{2}$

Organising pneumonia, acute fibrinous $\mathrm{OP}$, and diffuse alveolar damage are histological manifestations arising from a broad range of pulmonary insults. Temporal and regional heterogeneity of the pulmonary parenchymal alterations may result in diverse or mixed patterns upon lung biopsy sampling. Corticosteroids remain the first-line therapy in OP. Around two thirds of patients with OP respond to treatment with corticosteroids. However, the role of corticosteroids in diffuse alveolar damage remains controversial. The steroid response of acute fibrinous $\mathrm{OP}$ is intermediate, between that of diffuse alveolar damage and OP. ${ }^{3}$

To the best of our knowledge, few cases of postinfluenza OP have been reported in the literature. ${ }^{4,5}$ Most were influenza A infection and patients usually presented with refractory respiratory failure, incomplete recovery, or new radiological infiltrate after initial improvement. Most cases responded well to corticosteroid therapy.

The present case demonstrates that OP can complicate influenza-related ARDS. Physicians should be aware of this possibility: timely 
confirmation by histological proof, exclusion of superimposed nosocomial pneumonia, and initiation of corticosteroid therapy after balancing the risks and benefits may result in a more favourable outcome in the disease trajectory.

\section{Author contributions}

All authors contributed to the concept of study, acquisition and analysis of data, drafting of the manuscript, and had critical revision of the manuscript for important intellectual content. All authors had full access to the data, contributed to the study, approved the final version for publication, and take responsibility for its accuracy and integrity.

\section{Conflicts of interest}

All authors have disclosed no conflicts of interest.

\section{Funding/support}

This case report received no specific grant from any funding agency in the public, commercial, or not-for-profit sectors.

\section{Ethics approval}

The patient was treated in accordance with the Declaration of Helsinki. The patient verbally agreed publication of this anonymous case report.

\section{References}

1. Butnor KJ. Avoiding underdiagnosis, overdiagnosis, and misdiagnosis of lung carcinoma. Arch Pathol Lab Med 2008;132:1118-32.

2. Beasley MB. The pathologist's approach to acute lung injury. Arch Pathol Lab Med 2010;134:719-27.

3. Bihari S, Bailey M, Bersten AD. Steroids in ARDS: to be or not to be. Intensive Care Med 2016;42:931-3.

4. Cornejo R, Llanos $\mathrm{O}$, Fernández $\mathrm{C}$, et al. Organizing pneumonia in patients with severe respiratory failure due to novel A (H1N1) influenza. BMJ Case Rep 2010; 2010. pii: bcr0220102708.

5. Asai N, Yokoi T, Nishiyama N, et al. Secondary organizing pneumonia following viral pneumonia caused by severe influenza B: a case report and literature reviews. BMC Infect Dis 2017;17:572. 